Special Focus on Modeling, Analysis and Control of Stochastic Systems

\title{
Almost sure stabilization of hybrid systems by feedback control based on discrete-time observations of mode and state
}

\author{
Gongfei SONG ${ }^{1}$, Zhenyu LU², Bo-Chao ZHENG ${ }^{1}$ \& Xuerong $\mathrm{MAO}^{3 *}$ \\ ${ }^{1}$ Jiangsu Collaborative Innovation Center on Atmospheric Environment and Equipment Technology, \\ School of Information and Control, Nanjing University of Information Science and Technology, \\ Nanjing 210044, China; \\ ${ }^{2}$ School of Electronic and Information Engineering, Nanjing University of Information Science and Technology, \\ Nanjing 210044, China; \\ ${ }^{3}$ Department of Mathematics and Statistics, University of Strathclyde, Glasgow G1 1XH, UK
}

Received 22 August 2017/Accepted 27 October 2017/Published online 13 June 2018

\begin{abstract}
Although the mean square stabilization of hybrid systems by feedback control based on discretetime observations of state and mode has been studied by several authors since 2013, the corresponding almost sure stabilization problem has received little attention. Recently, Mao was the first to study the almost sure stabilization of a given unstable system $\dot{x}(t)=f(x(t))$ by a linear discrete-time stochastic feedback control $A x([t / \tau] \tau) \mathrm{d} B(t)$ (namely the stochastically controlled system has the form $\mathrm{d} x(t)=f(x(t)) \mathrm{d} t+$ $A x([t / \tau] \tau) \mathrm{d} B(t))$, where $B(t)$ is a scalar Brownian, $\tau>0$, and $[t / \tau]$ is the integer part of $t / \tau$. In this paper, we consider a much more general problem. That is, we study the almost sure stabilization of a given unstable hybrid system $\dot{x}(t)=f(x(t), r(t))$ by nonlinear discrete-time stochastic feedback control $u(x([t / \tau] \tau), r([t / \tau] \tau)) \mathrm{d} B(t)$ (so the stochastically controlled system is a hybrid stochastic system of the form $\mathrm{d} x(t)=f(x(t), r(t)) \mathrm{d} t+u(x([t / \tau] \tau), r([t / \tau] \tau)) \mathrm{d} B(t))$, where $B(t)$ is a multi-dimensional Brownian motion and $r(t)$ is a Markov chain.

Keywords Brownian motion, Markov chain, generalized Itô formula, almost sure exponential stability, stochastic feedback control

Citation Song G F, Lu Z Y, Zheng B-C, et al. Almost sure stabilization of hybrid systems by feedback control based on discrete-time observations of mode and state. Sci China Inf Sci, 2018, 61(7): 070213, https://doi.org/10. $1007 / \mathrm{s} 11432-017-9297-1$
\end{abstract}

\section{Introduction}

In recent years, stochastic systems have been considered by many researchers since many practical systems can be modeled using these kinds of systems. Many significant results for stochastic systems have been reported (see [1-13]). Markovian jump systems are a special class of hybrid stochastic systems, which can be found in some engineering systems including power systems, manufacturing systems, ecosystems, and so forth. The literature in this area is huge and lots of papers are open access, thus we only mention a few [14-18]. Shaikhet [19] provided the sufficient conditions of asymptotic mean square stability for Markovian systems with delay. Mao [20] discussed the problem of exponential stability of general nonlinear Markovian jump systems.

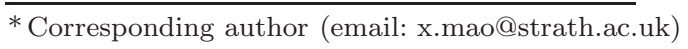


As is well known, a given unstable system can be stabilized by noise or noise can be used to make a system more stable when it is already stable. Arnold et al. [21] pointed out that a linear system can be stabilized by zero mean stationary parameter noise. In [22], a linear hybrid stochastic system was stabilized by Gaussian type noise. In addition, Khasminskii [23] proposed that a system was stabilized by using two types of white noise. It was shown in [24] that an unstable nonlinear system can be stabilized by Brownian motion provided the growth condition is linear. Mao [25] showed that any nonlinear system $\dot{x}(t)=f(x(t), t)$ whose coefficient satisfied the condition $|f(x, t)| \leqslant K|x|, K>0$, it was possible to use the Brownian motions to stabilize the system. It is worth noting that Appleby et al. [26] presented a general theory on the problem of stochastic stabilization for a nonlinear functional differential equation by noise. Mao et al. [27] developed an unstable Markovian jump system $\dot{x}(t)=f(x(t), r(t), t)$ that can be stabilized by stochastic control and the partial subsystem was controlled. In other words, the space $\mathbb{S}$ of the Markov chain was divided into two proper subspaces $\mathbb{S}_{1}$ and $\mathbb{S}_{2}$, i.e., $\mathbb{S}=\mathbb{S}_{1} \cup \mathbb{S}_{2}$. In summary, Mao et al. [27] considered the controlled stochastic system

$$
\mathrm{d} x(t)=f(x(t), r(t), t) \mathrm{d} t+u(r(t), t) \mathrm{d} B(t),
$$

where $u(i, t)=0$ for $i \in \mathbb{S}_{1}$ while $u(i, t)=u(i, x(t))$ was a feedback control for $i \in \mathbb{S}_{2}$. New methods and sufficient conditions on the stochastic stabilization for Markovian jump systems were provided in [28]. With some applications, two examples on stabilization and destabilization by noise in the plane were presented in [29].

We should of course point out that the corresponding problem based on discrete-time state observations has already been studied by some authors. Recently, Mao [30] was the first to study this stabilization problem. He also obtained a bound $\tau^{*}$ on $\tau$ for the controlled system to be stable as long as $\tau<\tau^{*}$ (plus some other conditions of course). Here $\tau>0$ is the duration between two consecutive observations. From the point of control cost, it is clearly better to have a larger $\tau^{*}$. Influenced by [30], a number of recent papers (e.g., $[31,32]$ ) have significantly improved the bound $\tau^{*}$. Mao et al. [31] established a better bound on $\tau^{*}$ by considering a couple of important classes of hybrid stochastic systems and using their special features. On the other hand, a better bound on $\tau^{*}$ was also obtained in [32] by making use of Lyapunov functionals. In particular, Song et al. [33] pointed out that the discrete-time feedback control in controlled hybrid stochastic systems was based on not only the discrete-time observations of the state, $x(k \tau)(k=0,1,2, \ldots)$ but also it was still dependent on the discrete-time observations of the mode, $r(k \tau)$, on $k=0,1,2, \ldots$.

Observing that all the papers mentioned above were concerned with the mean square stabilization by the discrete-time feedback control in the drift part, Mao [34] discussed the following almost sure exponential stabilization by discrete-time feedback control in the diffusion part. Given an unstable nonlinear system $\dot{x}(t)=f(x(t))$, Mao designed a feedback control $A x([t / \tau] \tau)$, based on the discrete-time state observations, in the diffusion part so that the corresponding closed-loop system

$$
\mathrm{d} x(t)=f(x(t)) \mathrm{d} t+A x([t / \tau] \tau) \mathrm{d} B(t)
$$

was almost surely exponentially stable. Here $B(t)$ was a scalar Brownian motion, $f: \mathbb{R}^{n} \rightarrow \mathbb{R}^{n}$ satisfied

$$
|f(x)-f(y)| \leqslant \alpha|x-y|, \quad \forall x, y \in \mathbb{R}^{n},
$$

for some $\alpha>0$ and $f(0)=0$, and $A$ was an $n \times n$ real-valued matrix such that

$$
|A x|^{2} \leqslant \rho_{1}|x|^{2} \quad \text { and } \quad\left|x^{\mathrm{T}} A x\right|^{2} \geqslant \rho_{2}|x|^{2}, \quad \forall x \in \mathbb{R}^{n},
$$

for some positive numbers $\rho_{1}$ and $\rho_{2}$ satisfying $\rho_{2}-0.5 \rho_{1}>\alpha$. Mao [34] showed that there was a positive number $\tau^{*}$ such that the controlled system (2) was almost surely exponentially stable provided that $\tau<\tau^{*}$. To the best of the authors' knowledge, the problem of almost sure exponential stabilization for hybrid stochastic systems has received little attention, in particular, in the framework of stochastic feedback control based on discrete-time observations of mode and state. 
These motivate us to consider the following more general problem: if the given unstable system is expressed as a hybrid stochastic system $\dot{x}(t)=f(x(t), r(t))$, can we design a discrete-time feedback control $u(x([t / \tau] \tau), r([t / \tau] \tau))$, based on the discrete-time observations of both state and mode, in the diffusion part so that the following closed-loop system

$$
\mathrm{d} x(t)=f(x(t), r(t)) \mathrm{d} t+u(x([t / \tau] \tau), r([t / \tau] \tau)) \mathrm{d} B(t)
$$

is almost surely exponentially stable? Here $B(t)$ is an $m$-dimensional Brownian motion, $r(t)$ is a Markov chain in a finite state space $\mathbb{S}$ while $f: \mathbb{R}^{n} \times \mathbb{S} \rightarrow \mathbb{R}^{n}$ and $u: \mathbb{R}^{n} \times \mathbb{S} \rightarrow \mathbb{R}^{n \times m}$. We highlight a number of key features.

- The stabilization is in the sense of almost sure exponential stability and there is far less known about this than the mean square stabilization.

- The control $u$ is in the diffusion part while it is nonlinear and $B(t)$ is multi-dimensional.

- The controlled system is a hybrid stochastic delay system.

- The discrete-time feedback control $u(x([t / \tau] \tau), r([t / \tau] \tau))$ is based on the discrete-time observations of both state and mode.

Let us begin to investigate this more general stabilization problem.

\section{Preliminaries and notation}

Throughout this paper, the notation is fairly standard. Here $|\cdot|$ denotes the Euclidean norm in $\mathbb{R}^{n}$. For a vector or matrix $A, A^{\mathrm{T}}$ denotes its transpose and $|A|=\sqrt{\operatorname{trace}\left(A^{\mathrm{T}} A\right)}$ represents the trace norm of matrix $A$. For a symmetric matrix $A, \lambda_{\max }(A)$ and $\lambda_{\min }(A)$ represent the largest and smallest eigenvalue, respectively. Here $\left(\Omega, \mathcal{F},\left\{\mathcal{F}_{t}\right\}_{t \geqslant 0}, \mathbb{P}\right)$ is a complete probability space, where $\left\{\mathcal{F}_{t}\right\}_{t \geqslant 0}$ satisfies the conditions that it is right continuous and $\mathcal{F}_{0}$ contains all $\mathbb{P}$-null sets. In addition, we use $B(t), t \geqslant 0$, as an $m$-dimensional Brownian motion. The continuous-time Markov chain $r(t), t \geqslant 0$, takes discrete values in a given finite set $\mathbb{S}=\{1,2, \ldots, N\}$ and has the generator $\Gamma=\left(\gamma_{i j}\right)_{N \times N}$ given by

$$
\mathbb{P}\{r(t+\Delta)=j \mid r(t)=i\}= \begin{cases}\gamma_{i j} \Delta+o(\Delta), & \text { if } i \neq j, \\ 1+\gamma_{i i} \Delta+o(\Delta), & \text { if } i=j\end{cases}
$$

with $\Delta>0$ and $\gamma_{i i}=-\sum_{j \neq i} \gamma_{i j}$. Here $\gamma_{i j} \geqslant 0$ denotes the transition rate from $i$ to $j$. The notation $\pi=\left(\pi_{1}, \pi_{2}, \ldots, \pi_{N}\right) \in \mathbb{R}^{1 \times N}$ represents a stationary (probability) distribution. Furthermore, one can find the linear equation $\pi \Gamma=0$ subject to $\sum_{j=1}^{N} \pi_{j}=1$ and $\pi_{j}>0$ for all $j \in \mathbb{S}$. In particular, we recall that $-\gamma_{i i}=\sum_{j \neq i} \gamma_{i j}>0$. We state a lemma that estimates the probability of jumps.

Lemma 1. For any $t \geqslant 0, v>0$, and $i \in \mathbb{S}$,

$$
\mathbb{P}(r(s) \neq i, \text { for some } s \in[t, t+v] \mid r(t)=i) \leqslant 1-\mathrm{e}^{-\hat{\gamma} v},
$$

where

$$
\hat{\gamma}=\max _{i \in \mathbb{S}}\left(-\gamma_{i i}\right)
$$

To show this lemma, define the stopping time

$$
\zeta_{i}=\inf \{s \geqslant t: r(s) \neq i\} l,
$$

given $r(t)=i$, and let $\inf \emptyset=\infty$ where $\emptyset$ denotes the empty set as usual. It is well known (see [14]) that $\zeta_{i}-t$ has the exponential probability distribution with parameter $-\gamma_{i i}$. Hence,

$$
\begin{aligned}
& \mathbb{P}(r(s) \neq i \text { for some } s \in[t, t+v] \mid r(t)=i) \\
& \quad=\mathbb{P}\left(\zeta_{i}-t \leqslant v \mid r(t)=i\right)=\int_{0}^{v}-\gamma_{i i} \mathrm{e}^{\gamma_{i i} s} \mathrm{~d} s
\end{aligned}
$$




$$
\begin{aligned}
& \text { Song G F, et al. Sci China Inf Sci July } 2018 \text { Vol. } 61070213: 4 \\
& =1-\mathrm{e}^{\gamma_{i i} v} \leqslant 1-\mathrm{e}^{-\hat{\gamma} v}
\end{aligned}
$$

as desired.

Consider the following unstable hybrid stochastic system

$$
\dot{x}(t)=f(x(t), r(t)), \quad t \geqslant 0
$$

with the initial conditions $x(0)=x_{0} \in \mathbb{R}^{n}$ and $r(0)=r_{0} \in \mathbb{S}$, where $f: \mathbb{R}^{n} \times \mathbb{S} \rightarrow \mathbb{R}^{n}, x(t)$ is the state, and $r(t)$ is the mode. We are required to design a stochastic feedback control $u(x([t / \tau] \tau), r([t / \tau] \tau)) \mathrm{d} B(t)$ based on the observations of state $x([t / \tau] \tau)$ and mode $r([t / \tau] \tau)$ at the discrete times $0, \tau, 2 \tau, \ldots$ such that the corresponding closed-loop system

$$
\mathrm{d} x(t)=f(x(t), r(t)) \mathrm{d} t+u(x([t / \tau] \tau), r([t / \tau] \tau)) \mathrm{d} B(t), \quad t \geqslant 0
$$

becomes almost surely exponentially stable, where the positive constant $\tau>0$ denotes the duration between two consecutive observations, $[t / \tau]$ is the integer part of $t / \tau$, and $u: \mathbb{R}^{n} \times \mathbb{S} \rightarrow \mathbb{R}^{n \times m}$ is the control input. For the existence and uniqueness of the solution to the controlled system, we impose the following assumption.

Assumption 1. There exist two positive constants $K_{1}$ and $K_{2}$ such that

$$
|f(x, i)-f(y, i)| \leqslant K_{1}|x-y| \text { and }|u(x, i)-u(y, i)| \leqslant K_{2}|x-y|
$$

for all $(x, y, i) \in \mathbb{R}^{n} \times \mathbb{R}^{n} \times \mathbb{S}$.

This assumption guarantees that for any initial state $x(0)=x_{0} \in \mathbb{R}^{n}$ and mode $r(0)=r_{0} \in \mathbb{S}$, the controlled system (8) has a unique solution $x(t)$ on $t \in \mathbb{R}_{+}$and $\mathrm{E}|x(t)|^{2}<\infty$ for all $t \geqslant 0$. In fact, for $t \in[0, \tau]$, system $(8)$ becomes

$$
\mathrm{d} x(t)=f(x(t), r(t)) \mathrm{d} t+u(x(0), r(0)) \mathrm{d} B(t)
$$

with the initial state $x(0)=x_{0}$ and mode $r(0)=r_{0}$. It is easy to show (see [27, Theorem 3.13]) that this hybrid stochastic system has a unique solution $x(t)$ on $t \in[0, \tau]$ with $\mathrm{E}|x(t)|^{2}<\infty$. For $t \in[\tau, 2 \tau]$, system (8) becomes

$$
\mathrm{d} x(t)=f(x(t), r(t)) \mathrm{d} t+u(x(\tau), r(\tau)) \mathrm{d} B(t)
$$

with the initial state $x(\tau)$ and mode $r(\tau)$ at $t=\tau$. It is easy to see that this hybrid stochastic system has a unique solution $x(t)$ on $t \in[\tau, 2 \tau]$ with $\mathrm{E}|x(t)|^{2}<\infty$. Repeating this procedure, we can see what we have just claimed. Let us denote the solution by $x\left(t ; x_{0}, r_{0}\right)$. We see easily show that if $x_{0}=0$, then $x\left(t ; 0, r_{0}\right)=0$ for all $t \geqslant 0$ almost surely. This is known as the trivial solution.

The purpose of this paper is to find sufficient conditions on the coefficient $f$ and the control input $u$ as well as to obtain a positive bound $\tau^{*}$ such that the controlled system (8) is almost surely exponentially stable as long as $\tau \leqslant \tau^{*}$. By the almost sure exponential stability, we mean that

$$
\limsup \frac{1}{t} \log \left(\left|x\left(t ; x_{0}, r_{0}\right)\right|\right)<0 \quad \text { almost surely, }
$$

for any $\left(x_{0}, r_{0}\right) \in \mathbb{R}^{n} \times \mathbb{S}$ (see $\left.[7,8,23,25]\right)$. We also observe that when $\tau \rightarrow 0$, the controlled system (8) becomes the corresponding hybrid stochastic system

$$
\mathrm{d} y(t)=f(y(t), r(t)) \mathrm{d} t+u(y(t), r(t)) \mathrm{d} B(t)
$$

on $t \geqslant 0$ with the initial condition $(y(0), r(0))=\left(x_{0}, r_{0}\right)$. Under Assumption 1, system (10) has a unique solution (see $[17,20])$. Denote the unique solution by $y\left(t ; x_{0}, r_{0}\right)$ on $t \geqslant 0$. 


\section{Main results}

We see clearly from the discussion in the previous section that the conditions we need to impose should at least guarantee the almost sure exponential stability of the corresponding hybrid stochastic system (10). Although there are many useful criteria on the almost sure exponential stability, we use that established by [20]. Accordingly, we impose the following assumptions.

Assumption 2. For each $i \in \mathbb{S}$, there exist constant triples $\alpha_{i} \in \mathbb{R}, \rho_{i} \geqslant 0$, and $\sigma_{i} \geqslant 0$ such that

$$
x^{\mathrm{T}} f(x, i) \leqslant \alpha_{i}|x|^{2}, \quad|u(x, i)| \leqslant \rho_{i}|x|, \quad\left|x^{\mathrm{T}} u(x, i)\right| \geqslant \sigma_{i}|x|^{2},
$$

for all $x \in \mathbb{R}^{n}$. Set $\hat{\alpha}=\max _{i \in \mathbb{S}} \alpha_{i}$ and $\hat{\rho}=\max _{i \in \mathbb{S}} \rho_{i}$.

Assumption 3. There is a constant $p \in(0,1)$ such that the $N \times N$ matrix

$$
\mathcal{A}(p):=\operatorname{diag}\left(\theta_{1}(p), \ldots, \theta_{N}(p)\right)-\Gamma
$$

is a nonsingular $M$-matrix, where

$$
\theta_{i}(p):=\frac{p(2-p) \sigma_{i}^{2}}{2}-\frac{p \rho_{i}^{2}}{2}-p \alpha_{i}
$$

and $\alpha_{i}, \sigma_{i}, \rho_{i}$ are the constants specified in Assumption 2.

Let us make some comments on these assumptions. First, we point out that Assumptions 1 and 2 force

$$
f(0, i)=0 \quad \text { and } \quad u(0, i)=0, \quad \forall i \in \mathbb{S}
$$

which meet the stability purpose in this paper. In fact, $g(0, i)=0$ follows from the second inequality in (11). To show that $f(0, i)=0$ for all $i \in \mathbb{S}$, we assume otherwise that there were some $i \in \mathbb{S}$ such that $z:=f(0, i) \neq 0$. Choose a constant $b$ such that $0<b<1 /\left(K_{1}+\left|\alpha_{i}\right|\right)$ and let $x=b z$. Then, by the first inequality in (11),

$$
b z^{\mathrm{T}} f(b z, i) \leqslant \alpha_{i} b^{2}|z|^{2} \leqslant\left|\alpha_{i}\right| b^{2}|z|^{2} .
$$

On the other hand, by Assumption 1,

$$
b z^{\mathrm{T}} f(b z, i)=b|z|^{2}+b z^{\mathrm{T}}(f(b z, i)-f(0, i)) \geqslant b|z|^{2}-K_{1} b^{2}|z|^{2} .
$$

Hence,

$$
b|z|^{2}-K_{1} b^{2}|z|^{2} \leqslant\left|\alpha_{i}\right| b^{2}|z|^{2} .
$$

This implies that

$$
1 \leqslant\left(K_{1}+\left|\alpha_{i}\right|\right) b,
$$

but this is a contradiction as $b<1 /\left(K_{1}+\left|a_{i}\right|\right)$. We therefore must have (14). Recalling that $y\left(t ; x_{0}, r_{0}\right)$ denotes the solution of the hybrid stochastic system (10), we can hence highlight a significant property given in Mao [20, Lemma 2.1], which then leads to

$$
\mathbb{P}\left\{y\left(t ; x_{0}, r_{0}\right) \neq 0 \text { on } t \geqslant 0\right\}=1, \quad \forall x_{0} \neq 0 .
$$

That is, if any initial solution of system (10) is a nonzero state, almost all the trajectories of system (10) will never converge to the origin. Thus, Lyapunov functions can be chosen in a variety of ways.

We also emphasize that we are only interested in the case when $\hat{\alpha} \geqslant 0$ in this paper; otherwise, the given hybrid system (7) is already stable (see [28]) and there is no need to stabilize it using feedback control. We should also point out that we always have $\hat{\alpha} \leqslant K_{1}$ and $\hat{\rho} \leqslant K_{2}$, but we might have $\hat{\alpha}<K_{1}$ and $\hat{\rho}<K_{2}$ in many cases. For example, consider the scalar case where $f(x, i)=-x+a_{i} \sin (x)$ and $u(x, i)=x-b_{i} \sin (x)$ where $a_{i} \in[1,2], b_{i} \in(0,1]$, but $a_{1}=2$ and $b_{1}=1$. It is easy to see that $K_{1}=3$ and $K_{2}=2$. On the other hand, $x f(x, i)=-x^{2}+a_{i} \sin (x) x \leqslant\left(a_{i}-1\right)|x|^{2}$ so $\hat{\alpha}=1 \leqslant K_{1}$. Moreover, $0 \leqslant|u(x, i)| \leqslant|x|$ for $|x| \leqslant \pi$ whereas $0 \leqslant|u(x, i)| \leqslant|x|+b_{i} \leqslant\left(1+b_{i} / \pi\right)|x|$ for $|x|>\pi$ so we have 
$\hat{\rho}=(1+1 / \pi)<K_{2}$. We also observe that once Assumption 2 holds, the verification of Assumption 3 depends very much on the choice of $p \in(0,1)$. In Appendix A, we give some easier conditions that guarantee the existence of such a $p$ and, hence, for Assumption 3 to hold.

The following lemma shows that the corresponding hybrid stochastic system (10) is exponentially stable in the $p$ th moment and, hence, by [27, Theorem 5.9], the hybrid stochastic system is also almost surely exponentially stable.

Lemma 2. Let Assumptions 1-3 hold. Define

$$
\left(c_{1}, \ldots, c_{N}\right)^{\mathrm{T}}=\mathcal{A}^{-1}(p)(1, \ldots, 1)^{\mathrm{T}},
$$

so all $c_{i}$ are positive by the theory of $M$-matrices $[2,27]$ or by Lemma A1 in the Appendix A and let $c_{\min }=\min _{1 \leqslant i \leqslant N} c_{i}$ and $c_{\max }=\max _{1 \leqslant i \leqslant N} c_{i}$. Then the solution of the hybrid stochastic system (10) satisfies

$$
\mathrm{E}\left|y\left(t ; x_{0}, r_{0}\right)\right|^{p} \leqslant M\left|x_{0}\right|^{p} \mathrm{e}^{-\gamma t}, \quad \forall t \geqslant 0,
$$

for all $\left(x_{0}, r_{0}\right) \in \mathbb{R}^{n} \times \mathbb{S}$, where $\gamma=1 / c_{\max }$ and $M=c_{\max } / c_{\min }$.

Proof. For $x_{0}=0$, that is $y\left(t ; 0, r_{0}\right)=0$, we can deduce that the assertion is natural. Fix $x_{0} \neq 0$ and $r_{0} \in \mathbb{S}$ arbitrarily and write $y\left(t ; x_{0}, r_{0}\right)=y(t)$. Recalling (15), we have that $y(t) \neq 0$ for all $t \geqslant 0$ almost surely. Define the Lyapunov function

$$
V(y, t, i)=c_{i}|y|^{p} \mathrm{e}^{\gamma t}, \quad \text { for }(y, t, i) \in\left(\mathbb{R}^{n}-\{0\}\right) \times \mathbb{R}_{+} \times \mathbb{S} .
$$

We can therefore apply the generalized Itô formula (see [27, Theorem 1.45]) to obtain that

$$
\mathrm{E} V(y(t), t, r(t))=V\left(x_{0}, 0, r(0)\right)+\mathrm{E} \int_{0}^{t} L V(y(s), s, r(s)) \mathrm{d} s,
$$

for $t \geqslant 0$, where $L V:\left(\mathbb{R}^{n}-\{0\}\right) \times \mathbb{R}_{+} \times \mathbb{S} \rightarrow \mathbb{R}$ is defined by

$$
\begin{aligned}
L V(y, t, i)= & \mathrm{e}^{\gamma t}\left(\gamma c_{i}|y|^{p}+p c_{i}|y|^{p-2} y^{\mathrm{T}} f(y, i)+\frac{p c_{i}}{2}|y|^{p-2}|u(y, i)|^{2}\right. \\
& \left.-\frac{p(2-p) c_{i}}{2}|y|^{p-4}\left|y^{\mathrm{T}} u(y, i)\right|^{2}+\sum_{j=1}^{N} \gamma_{i j} c_{j}|y|^{p}\right) .
\end{aligned}
$$

By Assumption 2 and then using definition (13) of $\theta_{i}(p)$, we have

$$
L V(y, t, i) \leqslant \mathrm{e}^{\gamma t}|y|^{p}\left(1-c_{i} \theta_{i}(p)+\sum_{j=1}^{N} \gamma_{i j} c_{j}\right) .
$$

However, by (16) and (12),

$$
c_{i} \theta_{i}(p)-\sum_{j=1}^{N} \gamma_{i j} c_{j}=1, \quad \forall i \in \mathbb{S} .
$$

Hence, we have

$$
L V(y, t, i) \leqslant 0
$$

Substituting this into (18) yields

$$
\mathrm{E} V(y(t), t, r(t)) \leqslant V\left(x_{0}, 0, r(0)\right) .
$$

This implies

$$
c_{\min } \mathrm{e}^{\gamma t} \mathrm{E}|y(t)|^{p} \leqslant c_{\max }\left|x_{0}\right|^{p},
$$

which is the desired assertion (17).

To simplify our notation, we let $\delta_{t}=[t / \tau] \tau$ for $t \geqslant 0$ and set $t_{k}=k \tau$ for $k=0,1,2, \ldots$ from now on. 
Lemma 3. Let Assumptions 1 and 2 hold. Then for any initial condition $\left(x_{0}, r_{0}\right) \in \mathbb{R}^{n} \times \mathbb{S}$,

$$
\mathrm{E}\left|x\left(t ; x_{0}, r_{0}\right)\right|^{2} \leqslant\left|x_{0}\right|^{2} \mathrm{e}^{\left(2 \hat{\alpha}+\hat{\rho}^{2}\right) t}
$$

and

$$
\mathrm{E}\left|x\left(t ; x_{0}, r_{0}\right)-x\left(\delta_{t} ; x_{0}, r_{0}\right)\right|^{2} \leqslant 2 \tau\left(K_{1}^{2} \tau+\hat{\rho}^{2}\right)\left|x_{0}\right|^{2} \mathrm{e}^{\left(2 \hat{\alpha}+\hat{\rho}^{2}\right) t} \geqslant 0
$$

for all $t \geqslant 0$.

Proof. Fix any $\left(x_{0}, r_{0}\right) \in \mathbb{R}^{n} \times \mathbb{S}$ and write $x\left(t ; x_{0}, r_{0}\right)=x(t)$. By the Itô formula and Assumption 2, it follows that

$$
\mathrm{E}|x(t)|^{2} \leqslant\left|x_{0}\right|^{2}+\mathrm{E} \int_{0}^{t}\left(2 \hat{\alpha}|x(s)|^{2}+\hat{\rho}^{2}\left|x\left(\delta_{s}\right)\right|^{2}\right) \mathrm{d} s \leqslant\left|x_{0}\right|^{2}+\left(2 \hat{\alpha}+\hat{\rho}^{2}\right) \int_{0}^{t}\left(\sup _{0 \leqslant u \leqslant s} \mathrm{E}|x(u)|^{2}\right) \mathrm{d} s,
$$

for $t \geqslant 0$. As the second term on the right-hand side is increasing in $t$, we can obtain

$$
\sup _{0 \leqslant u \leqslant t} \mathrm{E}|x(t)|^{2} \leqslant\left|x_{0}\right|^{2}+\left(2 \hat{\alpha}+\hat{\rho}^{2}\right) \int_{0}^{t}\left(\sup _{0 \leqslant u \leqslant s} \mathrm{E}|x(u)|^{2}\right) \mathrm{d} s,
$$

which implies the desire assertion (19) by the well-known Gronwall inequality. Moreover, by Assumptions 1 and 2, we further derive that

$$
\mathrm{E}\left|x(t)-x\left(\delta_{t}\right)\right|^{2} \leqslant 2 \tau K_{1}^{2} \int_{\delta_{t}}^{t} \mathrm{E}|x(s)|^{2} \mathrm{~d} s+2 \tau \hat{\rho}^{2} \mathrm{E}\left|x\left(\delta_{t}\right)\right|^{2} .
$$

This, together with (19), implies another assertion (20).

Lemma 4. Let Assumptions 1 and 2 hold and $p \in(0,1)$. Then for any initial condition $\left(x_{0}, r_{0}\right) \in \mathbb{R}^{n} \times \mathbb{S}$,

$$
\mathrm{E}\left|x\left(t ; x_{0}, r_{0}\right)-y\left(t ; x_{0}, r_{0}\right)\right|^{p} \leqslant\left|x_{0}\right|^{p} \mathrm{e}^{p\left(K_{1}+1.5 K_{2}^{2}\right) t}\left(H(\tau)\left[\mathrm{e}^{\left(2 \hat{\alpha}+\hat{\rho}^{2}\right) t}-1\right]\right)^{p / 2},
$$

for all $t \geqslant 0$, where

$$
H(\tau)=\frac{6 K_{2}^{2}\left[\tau\left(K_{1}^{2} \tau+\hat{\rho}^{2}\right)+2\left(1-\mathrm{e}^{-\hat{\gamma} \tau}\right)\right]}{2 \hat{\alpha}+\hat{\rho}^{2}} .
$$

Proof. Fix any $\left(x_{0}, r_{0}\right) \in \mathbb{R}^{n} \times \mathbb{S}$ and set $x\left(t ; x_{0}, r_{0}\right)=x(t)$ and $y\left(t ; x_{0}, r_{0}\right)=y(t)$. By applying the Itô formula and Assumption 1, it can be verified that

$$
\mathrm{E}|x(t)-y(t)|^{2} \leqslant 2 K_{1} \mathrm{E} \int_{0}^{t}|x(s)-y(s)|^{2} \mathrm{~d} s+J_{1}(t),
$$

for $t \geqslant 0$, where

$$
\begin{aligned}
J_{1}(t)= & \mathrm{E} \int_{0}^{t}\left|u\left(x\left(\delta_{s}\right), r\left(\delta_{s}\right)\right)-u(y(s), r(s))\right|^{2} \mathrm{~d} s \\
\leqslant & 3 \mathrm{E} \int_{0}^{t}\left(\left|u\left(x\left(\delta_{s}\right), r\left(\delta_{s}\right)\right)-u\left(x\left(\delta_{s}\right), r(s)\right)\right|^{2}\right. \\
& +\left|u\left(x\left(\delta_{s}\right), r(s)\right)-u(x(s), r(s))\right|^{2} \\
& \left.+|u(x(s), r(s))-u(y(s), r(s))|^{2}\right) \mathrm{d} s \\
\leqslant & 3 \mathrm{E} \int_{0}^{t}\left(\left|u\left(x\left(\delta_{s}\right), r\left(\delta_{s}\right)\right)-u\left(x\left(\delta_{s}\right), r(s)\right)\right|^{2}\right. \\
& \left.+K_{2}^{2}\left|x\left(\delta_{s}\right)-x(s)\right|^{2}+K_{2}^{2}|x(s)-y(s)|^{2}\right) \mathrm{d} s
\end{aligned}
$$

Substituting this into (22) yields

$$
\mathrm{E}|x(t)-y(t)|^{2} \leqslant\left(2 K_{1}+3 K_{2}^{2}\right) \int_{0}^{t} \mathrm{E}|x(s)-y(s)|^{2} \mathrm{~d} s+3 K_{2}^{2} J_{2}(t)+3 J_{3}(t),
$$


where

$$
\begin{gathered}
J_{2}(t)=\int_{0}^{t} \mathrm{E}\left|x\left(\delta_{s}\right)-x(s)\right|^{2} \mathrm{~d} s \\
J_{3}(t)=\int_{0}^{t} \mathrm{E}\left|u\left(x\left(\delta_{s}\right), r\left(\delta_{s}\right)\right)-u\left(x\left(\delta_{s}\right), r(s)\right)\right|^{2} \mathrm{~d} s .
\end{gathered}
$$

However, by Lemma 3,

$$
J_{2}(t) \leqslant \frac{2 \tau\left(K_{1}^{2} \tau+\hat{\rho}^{2}\right)\left|x_{0}\right|^{2}}{2 \hat{\alpha}+\hat{\rho}^{2}}\left[\mathrm{e}^{\left(2 \hat{\alpha}+\hat{\rho}^{2}\right) t}-1\right]
$$

To estimate $J_{3}(t)$, we let $\kappa=\kappa(t)=[t / \tau]$. Then

$$
J_{3}(t)=\sum_{k=0}^{\kappa} \int_{t_{k}}^{t \wedge t_{k+1}} \mathrm{E}\left|u\left(x\left(\delta_{s}\right), r\left(\delta_{s}\right)\right)-u\left(x\left(\delta_{s}\right), r(s)\right)\right|^{2} \mathrm{~d} s .
$$

By Assumption 2, we can derive that, for $t_{k} \leqslant s \leqslant t \wedge t_{k+1}$,

$$
\begin{aligned}
\mathrm{E}\left|u\left(x\left(\delta_{s}\right), r\left(\delta_{s}\right)\right)-u\left(x\left(\delta_{s}\right), r(s)\right)\right|^{2} \\
=\mathrm{E}\left|u\left(x\left(t_{k}\right), r\left(t_{k}\right)\right)-u\left(x\left(t_{k}\right), r(s)\right)\right|^{2} \\
=\mathrm{E}\left[\mathrm{E}\left(\left|u\left(x\left(t_{k}\right), r\left(t_{k}\right)\right)-u\left(x\left(t_{k}\right), r(s)\right)\right|^{2} \mid \mathcal{F}_{t_{k}}\right)\right] \\
\quad \leqslant \mathrm{E}\left[4 \hat{\rho}^{2}\left|x\left(t_{k}\right)\right|^{2} \mathrm{E}\left(I_{\left\{r(s) \neq r\left(t_{k}\right)\right\}} \mid \mathcal{F}_{t_{k}}\right)\right] \\
=\mathrm{E}\left[4 \hat{\rho}^{2}\left|x\left(t_{k}\right)\right|^{2} \mathrm{E}\left(\sum_{i \in \mathbb{S}} I_{\left\{r\left(t_{k}\right)=i\right\}} I_{\{r(s) \neq i\}} \mid \mathcal{F}_{t_{k}}\right)\right] \\
=\mathrm{E}\left[4 \hat{\rho}^{2}\left|x\left(t_{k}\right)\right|^{2} \sum_{i \in \mathbb{S}} I_{\left\{r\left(t_{k}\right)=i\right\}} \mathbb{P}\left(r(s) \neq i \mid r\left(t_{k}\right)=i\right)\right] .
\end{aligned}
$$

However, by Lemma 1,

$$
\begin{aligned}
& \mathbb{P}\left(r(s) \neq i \mid r\left(t_{k}\right)=i\right) \\
& \quad \leqslant \mathbb{P}\left(r(\bar{s}) \neq i \text { for some } \bar{s} \in\left[t_{k}, t \wedge t_{k+1}\right] \mid r\left(t_{k}\right)=i\right) \\
& \quad \leqslant 1-\mathrm{e}^{-\hat{\gamma} \tau}
\end{aligned}
$$

Hence,

$$
\mathrm{E}\left|u\left(x\left(\delta_{s}\right), r\left(\delta_{s}\right)\right)-u\left(x\left(\delta_{s}\right), r(s)\right)\right|^{2} \leqslant \mathrm{E}\left[4 \hat{\rho}^{2}\left|x\left(t_{k}\right)\right|^{2}\left(1-\mathrm{e}^{-\hat{\gamma} \tau}\right)\right]=4 \hat{\rho}^{2}\left(1-\mathrm{e}^{-\hat{\gamma} \tau}\right) \mathrm{E}\left|x\left(t_{k}\right)\right|^{2} .
$$

Substituting this into (25), we obtain

$$
J_{3}(t) \leqslant 4 \hat{\rho}^{2}\left(1-\mathrm{e}^{-\hat{\gamma} \tau}\right) \sum_{k=0}^{\kappa} \int_{t_{k}}^{t \wedge t_{k+1}} \mathrm{E}\left|x\left(t_{k}\right)\right|^{2} \mathrm{~d} s .
$$

However, by Lemma 3, we then have

$$
\begin{aligned}
\sum_{k=0}^{\kappa} \int_{t_{k}}^{t \wedge t_{k+1}} \mathrm{E}\left|x\left(t_{k}\right)\right|^{2} \mathrm{~d} s & \leqslant \sum_{k=0}^{\kappa} \int_{t_{k}}^{t \wedge t_{k+1}}\left|x_{0}\right|^{2} \mathrm{e}^{\left(2 \hat{\alpha}+\hat{\rho}^{2}\right) t_{k}} \mathrm{~d} s \\
& \leqslant \sum_{k=0}^{\kappa} \int_{t_{k}}^{t \wedge t_{k+1}}\left|x_{0}\right|^{2} \mathrm{e}^{\left(2 \hat{\alpha}+\hat{\rho}^{2}\right) s} \mathrm{~d} s=\left|x_{0}\right|^{2} \int_{0}^{t} \mathrm{e}^{\left(2 \hat{\alpha}+\hat{\rho}^{2}\right) s} \mathrm{~d} s \\
& =\frac{\left|x_{0}\right|^{2}}{2 \hat{\alpha}+\hat{\rho}^{2}}\left[\mathrm{e}^{\left(2 \hat{\alpha}+\hat{\rho}^{2}\right) t}-1\right] .
\end{aligned}
$$


Putting this into (28) gives

$$
J_{3}(t) \leqslant \frac{4 \hat{\rho}^{2}\left(1-\mathrm{e}^{-\hat{\gamma} \tau}\right)\left|x_{0}\right|^{2}}{2 \hat{\alpha}+\hat{\rho}^{2}}\left[\mathrm{e}^{\left(2 \hat{\alpha}+\hat{\rho}^{2}\right) t}-1\right] .
$$

Substituting (24) and (29) into (23), we obtain

$$
\mathrm{E}|x(t)-y(t)|^{2} \leqslant\left(2 K_{1}+3 K_{3}^{2}\right) \int_{0}^{t} \mathrm{E}|x(s)-y(s)|^{2} \mathrm{~d} s+\left|x_{0}\right|^{2} H(\tau)\left[\mathrm{e}^{\left(2 \hat{\alpha}+\hat{\rho}^{2}\right) t}-1\right] .
$$

Using the well-known Gronwall inequality, we have

$$
\mathrm{E}|x(t)-y(t)|^{2} \leqslant\left|x_{0}\right|^{2} H(\tau) \mathrm{e}^{\left(2 K_{1}+3 K_{3}^{2}\right) t}\left[\mathrm{e}^{\left(2 \hat{\alpha}+\hat{\rho}^{2}\right) t}-1\right] .
$$

Finally, we can obtain the desired assertion (21) by applying the Hölder inequality.

Lemma 5. Let Assumptions 1-3 hold. Choose a free parameter $\varepsilon \in(0,1)$. Let $\bar{\tau}>0$ be the unique root to the equation

$$
\mathrm{e}^{p\left(K_{1}+1.5 K_{2}^{2}\right)(\tau+\log (M / \varepsilon) / \gamma)}\left(H(\tau)\left[\mathrm{e}^{\left(2 \hat{\alpha}+\hat{\rho}^{2}\right)(\tau+\log (M / \varepsilon) / \gamma)}-1\right]\right)^{p / 2}=1-\varepsilon,
$$

where $H(\tau)$ and $\gamma, M$ have been given in Lemmas 2 and 4, respectively. Then, for each $\tau \in(0, \bar{\tau}]$, there exists a pair of positive integer $\bar{\kappa}$ and number $\lambda$ such that, for every initial condition $\left(x_{0}, r_{0}\right) \in \mathbb{R}^{n} \times \mathbb{S}$, the solution of system (8) satisfies

$$
\mathrm{E}\left|x\left(k \bar{\kappa} \tau ; x_{0}, r_{0}\right)\right|^{p} \leqslant\left|x_{0}\right|^{p} \mathrm{e}^{-\lambda k \bar{\kappa} \tau}, \quad \forall k=1,2,3, \ldots
$$

Proof. It is easy to see that the term on the left-hand side of (31) is a continuous increasing function of $\tau \geqslant 0$ and is equal to zero when $\tau=0$ whereas it tends to infinity as $\tau \rightarrow \infty$, thus Eq. (31) must have a unique root $\bar{\tau}>0$. Fix $\tau \in(0, \bar{\tau}]$ and $\left(x_{0}, r_{0}\right) \in \mathbb{R}^{n} \times \mathbb{S}$ arbitrarily and write $x\left(k \tau ; x_{0}, r_{0}\right)=x_{k}$ for $k=0,1,2, \ldots$. Let $\bar{\kappa}$ be the smallest positive integer that is no less than $\frac{\log (M / \varepsilon)}{\gamma \tau}$, namely

$$
\frac{\log (M / \varepsilon)}{\gamma \tau} \leqslant \bar{\kappa}<1+\frac{\log (M / \varepsilon)}{\gamma \tau}
$$

where $\gamma$ and $M$ have been defined in Lemma 2. This implies

$$
M \mathrm{e}^{-\gamma \bar{\kappa} \tau} \leqslant \varepsilon
$$

Write $y\left(\bar{\kappa} \tau ; x_{0}, r_{0}\right)=y_{\bar{\kappa}}$. By Lemma 2 ,

$$
\mathrm{E}\left|y_{\bar{\kappa}}\right|^{p} \leqslant M\left|x_{0}\right|^{p} \mathrm{e}^{-\gamma \bar{\kappa} \tau} \leqslant \varepsilon\left|x_{0}\right|^{p} .
$$

By the elementary inequality $(a+b)^{p} \leqslant a^{p}+b^{p}$ for any $a, b \geqslant 0$ and $0<p<1$, one can obtain

$$
\mathrm{E}\left|x_{\bar{\kappa}}\right|^{p} \leqslant \mathrm{E}\left|y_{\bar{\kappa}}\right|^{p}+\mathrm{E}\left|y_{\bar{\kappa}}-x_{\bar{\kappa}}\right|^{p} .
$$

Using (35) and Lemma 4, we obtain that

$$
\mathrm{E}\left|x_{\bar{\kappa}}\right|^{p} \leqslant\left|x_{0}\right|^{p}\left\{\varepsilon+\mathrm{e}^{p\left(K_{1}+1.5 K_{2}^{2}\right) \bar{\kappa} \tau}\left(H(\tau)\left[\mathrm{e}^{\left(2 \hat{\alpha}+\hat{\rho}^{2}\right) \bar{\tau}}-1\right]\right)^{p / 2}\right\} .
$$

Noting from (33) that $\bar{\kappa} \tau<\tau+\log (M / \varepsilon) / \gamma$, we have

$$
\begin{aligned}
\varepsilon & +\mathrm{e}^{p\left(K_{1}+1.5 K_{2}^{2}\right) \bar{\kappa} \tau}\left(H(\tau)\left[\mathrm{e}^{\left(2 \hat{\alpha}+\hat{\rho}^{2}\right) \bar{\tau}}-1\right]\right)^{p / 2} \\
& <\varepsilon+\mathrm{e}^{p\left(K_{1}+1.5 K_{2}^{2}\right)(\tau+\log (M / \varepsilon) / \gamma)}\left(H(\tau)\left[\mathrm{e}^{\left(2 \hat{\alpha}+\hat{\rho}^{2}\right)(\tau+\log (M / \varepsilon) / \gamma)}-1\right]\right)^{p / 2} \\
& \leqslant 1
\end{aligned}
$$


where Eq. (31) has been used. We may therefore write

$$
\varepsilon+\mathrm{e}^{p\left(K_{1}+1.5 K_{2}^{2}\right) \bar{\kappa} \tau}\left(H(\tau)\left[\mathrm{e}^{\left(2 \hat{\alpha}+\hat{\rho}^{2}\right) \bar{\tau}}-1\right]\right)^{p / 2}=\mathrm{e}^{-\lambda \bar{\kappa} \tau},
$$

for some $\lambda>0$. It then follows from (36) that

$$
\mathrm{E}\left|x_{\bar{\kappa}}\right|^{p} \leqslant\left|x_{0}\right|^{p} \mathrm{e}^{-\lambda \bar{\kappa} \tau} .
$$

Let us now discuss the solution $x(t)$ of hybrid stochastic system (8) on $t \geqslant \bar{\kappa} \tau$. This can be regarded as the solution of the hybrid stochastic system (8) with initial condition $\left(x_{\bar{\kappa}}, r(\bar{\kappa} \tau)\right)$ at time $t=\bar{\kappa} \tau$. Owing to the time-homogeneous property of hybrid stochastic system (8), we can thus easily show that

$$
\mathrm{E}\left(\left|x_{2 \bar{\kappa}}\right|^{p} \mid \mathcal{F}_{\bar{\kappa} \tau}\right) \leqslant\left|x_{\bar{\kappa}}\right|^{p} \mathrm{e}^{-\lambda \bar{\kappa} \tau} .
$$

This implies

$$
\mathrm{E}\left|x_{2 \bar{\kappa}}\right|^{p} \leqslant \mathrm{E}\left|x_{\bar{\kappa}}\right|^{p} \mathrm{e}^{-\lambda \bar{\kappa} \tau} \leqslant\left|x_{0}\right|^{p} \mathrm{e}^{-2 \lambda \bar{\kappa} \tau} .
$$

Repeating this procedure, we have

$$
\mathrm{E}\left|x_{k \bar{\kappa}}\right|^{p} \leqslant \mathrm{E}\left|x_{(k-1) \bar{\kappa}}\right|^{p} \mathrm{e}^{-\lambda \bar{\kappa} \tau} \leqslant\left|x_{0}\right|^{p} \mathrm{e}^{-\lambda k \bar{\kappa} \tau}, \quad \forall k=1,2,3, \ldots
$$

as desired. The proof is, hence, complete.

Now, we are in the position to present and prove our main results in this section.

Theorem 1. Let Assumptions 1-3 hold. Then there exists a positive number $\tau^{*}$ such that the stochastic controlled hybrid system (8) is almost surely exponentially stable provided $\tau \leqslant \tau^{*}$.

Proof. Choose a free parameter $\varepsilon \in(0,1)$. We note that the unique root of (31) is $\bar{\tau}>0$. Let $\tau^{*}=\bar{\tau}$. First, we set $\tau \in\left(0, \tau^{*}\right]$ and $\left(x_{0}, r_{0}\right) \in \mathbb{R} \times \mathbb{S}$, and then we write $x\left(t ; x_{0}, r_{0}\right)=x(t)$. In addition, let $\bar{\kappa}$ and $x_{k \bar{\kappa}}$ be the same as defined in the proof of Lemma 5 . For any $t \geqslant 0$, we can find a unique integer $k$ such that $t \in[k \bar{\kappa} \tau,(k+1) \bar{\kappa} \tau)$. By the time-homogeneous property of system (8), we see from Lemma 3 that

$$
\mathrm{E}\left(|x(t)|^{2} \mid \mathcal{F}_{k \bar{\kappa} \tau}\right) \leqslant\left|x_{k \bar{\kappa}}\right|^{2} \mathrm{e}^{\left(2 \hat{\alpha}+\hat{\rho}^{2}\right)(t-k \bar{\kappa} \tau)} \leqslant\left|x_{k \bar{\kappa}}\right|^{2} \mathrm{e}^{\left(2 \hat{\alpha}+\hat{\rho}^{2}\right) \bar{\kappa} \tau} .
$$

An application of the Hölder inequality yields

$$
\mathrm{E}\left(|x(t)|^{p} \mid \mathcal{F}_{k \bar{\kappa} \tau}\right) \leqslant C_{1}\left|x_{k \bar{\kappa}}\right|^{p},
$$

where $C_{1}=\mathrm{e}^{\left(\hat{\alpha}+0.5 \hat{\rho}^{2}\right) p \bar{\kappa} \tau}$. This, together with Lemma 5 , implies

$$
\mathrm{E}|x(t)|^{p} \leqslant C_{1} \mathrm{E}\left|x_{k \bar{\kappa}}\right|^{p} \leqslant C_{1}\left|x_{0}\right|^{p} \mathrm{e}^{-\lambda k \bar{\kappa} \tau} \leqslant C_{2}\left|x_{0}\right|^{p} \mathrm{e}^{-\lambda t},
$$

where $C_{2}=C_{1} \mathrm{e}^{\lambda \bar{\kappa} \tau}$. In other words, we have shown that the controlled system (8) is exponentially stable in the $p$ th moment. However, this is not yet what we require.

In the remainder of this proof, we show that this $p$ th moment exponential stability yields the almost sure exponential stability as desired. We should of course point out that [27, Theorem 5.9] shows this implication for hybrid stochastic systems, but our controlled system (8) is, in fact, a hybrid stochastic delay system. In the area of hybrid stochastic delay systems, Mao et al. [27, Theorem 7.24] showed this implication for $p \geqslant 1$, but here we have $p \in(0,1)$. Let $z$ be a positive integer sufficiently large for

$$
\left(\frac{\tau K_{1}}{z}\right)^{p} \leqslant 0.5
$$

Set $\varepsilon=\tau / z$. Let integers $k \geqslant 0$ and $0 \leqslant l \leqslant z-1$ be arbitrary. For $t \in\left[t_{k}+l \varepsilon, t_{k}+(l+1) \varepsilon\right]$, it follows from system (8) that

$$
x(t)=x\left(t_{k}+l \varepsilon\right)+\int_{t_{k}+l \varepsilon}^{t} f(x(s), r(s)) \mathrm{d} s+\int_{t_{k}+l \varepsilon}^{t} u\left(x\left(t_{k}\right), r\left(t_{k}\right)\right) \mathrm{d} B(s) .
$$


By the Burkholder-Davis-Gandy inequality (see [25]) and inequality (38), we then derive that

$$
\begin{aligned}
& \mathrm{E}\left(\sup _{t_{k}+l \varepsilon \leqslant t \leqslant t_{k}+(l+1) \varepsilon}|x(t)|^{p}\right) \\
& \leqslant \mathrm{E}\left|x\left(t_{k}+l \varepsilon\right)\right|^{p}+\mathrm{E}\left(\sup _{t_{k}+l \varepsilon \leqslant t \leqslant t_{k}+(l+1) \varepsilon}\left|\int_{t_{k}+l \varepsilon}^{t} f(x(s), r(s)) \mathrm{d} s\right|^{p}\right) \\
& \quad+\mathrm{E}\left(\sup _{t_{k}+l \varepsilon \leqslant t \leqslant t_{k}+(l+1) \varepsilon}\left|\int_{t_{k}+l \varepsilon}^{t} u\left(x\left(t_{k}\right), r\left(t_{k}\right)\right) \mathrm{d} B(s)\right|^{p}\right) \\
& \leqslant \mathrm{E}\left|x\left(t_{k}+l \varepsilon\right)\right|^{p}+\varepsilon^{p} K_{1}^{p} \mathrm{E}\left(\sup _{t_{k}+l \varepsilon \leqslant t \leqslant t_{k}+(l+1) \varepsilon}|x(t)|^{p}\right)+c_{p} \mathrm{E}\left(\varepsilon \hat{\rho}^{2}\left|x\left(t_{k}\right)\right|^{2}\right)^{p / 2} \\
& \leqslant C_{3} \mathrm{e}^{-\lambda t_{k}}+\varepsilon^{p} K_{1}^{p} \mathrm{E}\left(\sup _{t_{k}+l \varepsilon \leqslant t \leqslant t_{k}+(l+1) \varepsilon}|x(t)|^{p}\right),
\end{aligned}
$$

where $C_{3}=C_{2}\left|x_{0}\right|^{p}\left(1+c_{p} \varepsilon^{p / 2} \hat{\rho}^{p}\right)$ and $c_{p}$ is the constant from the Burkholder-Davis-Gandy inequality. By (39), we hence have

$$
\mathrm{E}\left(\sup _{t_{k}+l \varepsilon \leqslant t \leqslant t_{k}+(l+1) \varepsilon}|x(t)|^{p}\right) \leqslant 2 C_{3} \mathrm{e}^{-\lambda t_{k}} .
$$

Consequently,

$$
\begin{gathered}
\mathrm{E}\left(\sup _{t_{k} \leqslant t \leqslant t_{k+1}}|x(t)|^{p}\right)=\mathrm{E}\left(\max _{0 \leqslant l \leqslant z-1} \sup _{t_{k}+l \varepsilon \leqslant t \leqslant t_{k}+(l+1) \varepsilon}|x(t)|^{p}\right) \\
\leqslant \sum_{l=0}^{z-1} \mathrm{E}\left(\sup _{t_{k}+l \varepsilon \leqslant t \leqslant t_{k}+(l+1) \varepsilon}|x(t)|^{p}\right) \leqslant C_{4} \mathrm{e}^{-\lambda t_{k}},
\end{gathered}
$$

for all $k=0,1,2, \ldots$, where $C_{4}=2 z C_{3}$. This implies

$$
\mathbb{P}\left(\sup _{t_{k} \leqslant t \leqslant t_{k+1}}|x(t)|^{p} \geqslant \mathrm{e}^{-0.5 \lambda t_{k}}\right) \leqslant C_{4} \mathrm{e}^{-0.5 \lambda t_{k}},
$$

for all $i \geqslant 1$. The Borel-Cantelli lemma shows that, with probability 1 ,

$$
\sup _{t_{k} \leqslant t \leqslant t_{k+1}}|x(t)|^{p}<\mathrm{e}^{-0.5 \lambda t_{k}}
$$

holds for all but finitely many $k$. That is, for almost all $\omega \in \Omega$, there is an integer $k_{0}=k_{0}(\omega)$ such that

$$
\sup _{t_{k} \leqslant t \leqslant t_{k+1}}|x(t, \omega)|^{p}<\mathrm{e}^{-0.5 \lambda t_{k}}, \quad \forall k \geqslant k_{0}(\omega) .
$$

Therefore, for $t_{k} \leqslant t \leqslant t_{k+1}$ and $k \geqslant k_{0}$,

$$
\frac{1}{t} \log (|x(t, \omega)|)<-\frac{0.5 \lambda t_{k}}{p t_{k+1}}=-\frac{0.5 \lambda k}{p(k+1)} .
$$

Letting $t \rightarrow \infty$, we obtain

$$
\limsup _{t \rightarrow \infty} \frac{1}{t} \log (|x(t, \omega)|) \leqslant-\frac{\lambda}{2 p}
$$

for almost all $\omega \in \Omega$. This completes the proof.

\section{Design of linear feedback controls}

Consider the following unstable hybrid stochastic system

$$
\dot{x}(t)=f(x(t), r(t)), \quad t \geqslant 0,
$$


with the initial state $x(0)=x_{0} \in \mathbb{R}^{n}$ and mode $r(0)=r_{0} \in \mathbb{S}$, where $f: \mathbb{R}^{n} \times \mathbb{S} \rightarrow \mathbb{R}^{n}$. As before, we assume that $f$ meets conditions (9) and (11), namely there are constants $K_{1}>0$ and $\alpha_{i} \in \mathbb{R}(i \in \mathbb{S})$ such that

$$
|f(x, i)-f(y, i)| \leqslant K_{1}|x-y| \text { and } \quad x^{\mathrm{T}} f(x, i) \leqslant \alpha_{i}|x|^{2},
$$

for $x, y \in \mathbb{R}^{n}$ and $i \in \mathbb{S}$. Instead of nonlinear feedback controls, we are now looking for linear feedback controls. To avoid the notation becoming complicated, we set $B(t)$ be a scalar Brownian motion in this section (and leave the multi-dimensional case to the reader). The linear feedback control function we look for is of the form $u(x, i)=A(i) x$ so the controlled system becomes

$$
\mathrm{d} x(t)=f(x(t), r(t))+A(r[t / \tau]) x([t / \tau]) \mathrm{d} B(t),
$$

where $A(i) \in \mathbb{R}^{n \times n}$ for $i \in \mathbb{S}$ and we will often write $A(i)=A_{i}$. Noting that

$$
|u(x, i)-u(y, i)| \leqslant\left\|A_{i}\right\||x-y|, \quad \forall x, y \in \mathbb{R}^{n}, i \in \mathbb{S},
$$

we see that the second inequality in (9) holds with $K_{2}=\max _{i \in \mathbb{S}}\left\|A_{i}\right\|$.

\subsection{Observable in all modes}

We first consider the case where the state $x(t)$ is observable in every mode $i \in \mathbb{S}$. For each $i \in \mathbb{S}$, choose a matrix $D_{i} \in \mathbb{R}^{n \times n}$ such that

$$
\left\|D_{i}\right\|=1 \quad \text { and } \quad \lambda_{\min }\left(D_{i}+D_{i}^{\mathrm{T}}\right) \geqslant \sqrt{3} .
$$

Choose a nonnegative number $\delta_{i}$ such that

$$
\delta_{i}^{2}>4 \alpha_{i}
$$

Let $A_{i}=\delta_{i} D_{i}$. Noting that

$$
|u(x, i)|=\left|A_{i} x\right| \leqslant \delta_{i}|x| \quad \text { and } \quad\left|x^{\mathrm{T}} u(x, i)\right|=\left|x^{\mathrm{T}} A_{i} x\right|=\left.0.5 x^{\mathrm{T}}\left(A_{i}+A_{i}^{\mathrm{T}}\right) x\left|\geqslant \sqrt{3 / 4} \delta_{i}\right| x\right|^{2}
$$

for all $x \in \mathbb{R}^{n}$, we see that the 2 nd and 3rd inequality in (11) hold with $\rho_{i}=\delta_{i}$ and $\sigma_{i}=\sqrt{3 / 4} \delta_{i}$. By $(45)$,

$$
\sigma_{i}^{2}-0.5 \rho_{i}^{2}-\alpha_{i}=0.25 \delta_{i}^{2}-\alpha_{i}>0 .
$$

We can therefore find a $p \in(0,1)$ sufficiently small for

$$
\theta_{i}(p)=p\left(\frac{(2-p) \sigma_{i}^{2}}{2}-\frac{\rho_{i}^{2}}{2}-\alpha_{i}\right)>0, \quad \forall i \in \mathbb{S} .
$$

Consequently, recalling (12), we have

$$
\mathcal{A}(p) \mathbf{1}=\left(\theta_{1}(p), \ldots, \theta_{N}(p)\right)^{\mathrm{T}}>0,
$$

where $\mathbf{1}=(1, \ldots, 1)^{\mathrm{T}} \in \mathbb{R}^{N}$. By Lemma $\mathrm{A} 1, \mathcal{A}(p)$ is a nonsingular $M$-matrix. In other words, we have verified Assumption 3 under condition (45). In summary, we can conclude by using Theorem 1 that if we choose $D_{i}$ and $\delta_{i}$ to meet conditions (44) and (45), respectively, and let $A_{i}=\delta_{i} D_{i}$ for each $i \in \mathbb{S}$, there is a positive scalar $\tau^{*}$ such that the stochastic controlled hybrid system (43) is almost surely exponentially stable provided that $\tau \leqslant \tau^{*}$.

\subsection{Observable in some modes}

Let us now consider the case where the state of the underlying system is observable only for some modes but not all. To describe this situation, let us divide the space $\mathbb{S}$ of the Markov chain into two proper subspaces $\mathbb{S}_{1}$ and $\mathbb{S}_{2}$ (namely $\mathbb{S}=\mathbb{S}_{1} \cup \mathbb{S}_{2}$ and $\mathbb{S}_{1} \cap \mathbb{S}_{2}=\emptyset$ ). Assume that the state $x(t)$ is not observable when the system is in any mode $i \in \mathbb{S}_{1}$, but is fully observable in any mode $i \in \mathbb{S}_{2}$. Without any loss of generality, let us assume that $\mathbb{S}_{1}=\{1, \ldots, \bar{N}\}$ and $\mathbb{S}_{2}=\{\bar{N}+1, \ldots, N\}$ for some $1 \leqslant \bar{N}<N$. 
Let us now design our stochastic feedback control function $u(x, i)$. Given that the system is not observable in any mode $i \in \mathbb{S}_{1}$, it is reasonable to think it is not controllable in these modes so we must have $u(x, i)=0$ for $i \in \mathbb{S}_{1}$. For $i \in \mathbb{S}_{2}$, we seek the linear control function $u(x, i)=A_{i} x$ as in the last subsection. Hence, the controlled system can still be described by the hybrid system (43) as long as we set $A_{i}=0$ for $i \in \mathbb{S}_{1}$.

To design $A_{i}$ for $i \in \mathbb{S}_{2}$, we impose an additional condition that

$$
\text { for each } i \in \mathbb{S}_{1} \text {, there is a } j \in \mathbb{S}_{2} \text { such that } \gamma_{i j}>0 \text {. }
$$

In layman's terms, this condition means that given that the Markov chain is at state $i \in \mathbb{S}_{1}$ at any time $t$, it could jump to a state $j \in \mathbb{S}_{2}$ directly in very short time with a positive probability. Based on this condition, we can choose a pair of numbers $p \in(0,2 / 3)$ and $\beta \in(0,1)$ such that

$$
(1-\beta) \sum_{j=\bar{N}+1}^{N} \gamma_{i j}>p \alpha_{i}, \quad \forall i \in \mathbb{S}_{1} .
$$

We can then, for each $i \in \mathbb{S}_{2}$, find a nonnegative number $\delta_{i}$ such that

$$
\frac{\beta p \delta_{i}^{2}(2-3 p)}{8}>(1-\beta) \sum_{j=1}^{\bar{N}} \gamma_{i j}+\beta p \alpha_{i} .
$$

Choose a matrix $D_{i}$ satisfying condition (44) and let $A_{i}=\delta_{i} D_{i}$. We therefore see that the 2nd and 3rd inequality in (11) hold with $\rho_{i}=\delta_{i}$ and $\sigma_{i}=\sqrt{3 / 4} \delta_{i}$ for $i \in \mathbb{S}_{2}$ whereas $\rho_{i}=\sigma_{i}=0$ for $i \in \mathbb{S}_{1}$. Define

$$
\xi=(\overbrace{1, \cdots, 1}^{\bar{N} \text { times }}, \overbrace{\beta, \ldots, \beta}^{N-\bar{N} \text { times }})^{\mathrm{T}},
$$

and set

$$
\left(\zeta_{1}, \ldots, \zeta_{N}\right)^{\mathrm{T}}:=\mathcal{A}(p) \xi
$$

Then, for $i \in \mathbb{S}_{1}$,

$$
\zeta_{i}=-p \alpha_{i}-\sum_{j=1}^{\bar{N}} \gamma_{i j}-\beta \sum_{j=\bar{N}+1}^{N} \gamma_{i j}=-p \alpha_{i}+(1-\beta) \sum_{j=\bar{N}+1}^{N} \gamma_{i j}>0
$$

by (48), whereas for $i \in \mathbb{S}_{2}$,

$$
\begin{aligned}
\zeta_{i} & =\beta p\left(\frac{(2-3 p) \delta_{i}^{2}}{8}-\alpha_{i}\right)-\sum_{j=1}^{\bar{N}} \gamma_{i j}-\beta \sum_{j=\bar{N}+1}^{N} \gamma_{i j} \\
& =\beta p\left(\frac{(2-3 p) \delta_{i}^{2}}{8}-\alpha_{i}\right)-(1-\beta) \sum_{j=1}^{\bar{N}} \gamma_{i j} \\
& >0
\end{aligned}
$$

by (49). By Lemma $\mathrm{A} 1, \mathcal{A}(p)$ is a nonsingular $M$-matrix. In other words, we have to design $A_{i}$ to meet Assumption 3 under condition (47). We can therefore conclude by using Theorem 1 that if we design $A_{i}$ as described above, there is a positive scalar $\tau^{*}$ such that the stochastic controlled hybrid system (43) is almost surely exponentially stable provided that $\tau \leqslant \tau^{*}$.

\section{Conclusion}

Influenced by Mao [34], we have discussed the almost sure stabilization of a given unstable hybrid differential equation $\dot{x}(t)=f(x(t), r(t))$ by nonlinear discrete-time stochastic feedback control $u(x([t / \tau] \tau)$, 
$r([t / \tau] \tau)) \mathrm{d} B(t)$. We have shown that there is a positive number $\tau^{*}$ such that the stochastically controlled system $\mathrm{d} x(t)=f(x(t), r(t)) \mathrm{d} t+u(x([t / \tau] \tau), r([t / \tau] \tau)) \mathrm{d} B(t)$ is almost surely stable provided that $\tau<\tau *$ under the global Lipschitz condition plus the condition that guarantees the almost sure exponential stability of the corresponding hybrid stochastic system $\mathrm{d} x(t)=f(x(t), r(t)) \mathrm{d} t+u(x(t), r(t)) \mathrm{d} B(t)$. As a special but important case, we have discussed in more detail how to design the linear feedback controls.

Acknowledgements The authors would like to thank Leverhulme Trust (Grant No. RF-2015-385), Royal Society (Grant No. WM160014, Royal Society Wolfson Research Merit Award), Royal Society and Newton Fund (Grant No. NA160317, Royal Society-Newton Advanced Fellowship), Engineering and Physics Sciences Research Council (Grant No. EP/K503174/1), National Natural Science Foundation of China (Grant Nos. 61503190, 61473334, 61403207), Natural Science Foundation of Jiangsu Province (Grant Nos. BK20150927, BK20131000), and Ministry of Education (MOE) of China (Grant No. MS2014DHDX020) for their financial support. The first author would like to thank Chinese Scholarship Council for awarding him the scholarship to visit the University of Strathclyde for one year.

Open access This article is distributed under the terms of the Creative Commons Attribution License which permits any use, distribution, and reproduction in any medium, provided the original author(s) and source are credited.

\section{References}

1 Black F, Scholes M. The pricing of options and corporate liabilities. J Political Economy, 1973, 81: 637-654

2 Berman A, Plemmons R J. Nonnegative Matrices in the Mathematical Sciences. Philadelphia: Society for Industrial and Applied Mathematics, 1994

3 Krishnamurthy V, Wang X, Yin G. Spreading code optimization and adaptation in CDMA via discrete stochastic approximation. IEEE Trans Inform Theor, 2004, 50: 1927-1949

4 Luo Q, Gong Y Y, Jia C X. Stability of gene regulatory networks with Lévy noise. Sci China Inf Sci, 2017, 60: 072204

5 Luo Q, Shi L L, Zhang Y T. Stochastic stabilization of genetic regulatory networks. Neurocomputing, 2017, 266: $123-127$

6 Mao W H, Deng F Q, Wan A H. Robust $H_{2} / H_{\infty}$ global linearization filter design for nonlinear stochastic time-varying delay systems. Sci China Inf Sci, 2016, 59: 032204

7 Mao X R. Stability of Stochastic Differential Equations With Respect to Semimartingales. London: Longman Scientific and Technical, 1991

8 Mao X R. Exponential Stability of Stochastic Differential Equations. New York: Marcel Dekker, 1994

9 Tang M, Meng Q. Stochastic evolution equations of jump type with random coefficients: existence, uniqueness and optimal control. Sci China Inf Sci, 2017, 60: 118202

10 Willsky A S, Levy B C. Stochastic stability research for complex power systems. DOE Contract LIDS MIT Report No. ET-76-C-01-2295, 1979

11 Sworder D D, Rogers R O. An LQ-solution to a control problem associated with a solar thermal central receiver. IEEE Trans Automat Contr, 1983, 28: 971-978

12 Yin G, Liu R H, Zhang Q. Recursive algorithms for stock liquidation: a stochastic optimization approach. SIAM J Optim, 2002, 13: 240-263

13 Zhang Q. Stock trading: an optimal selling rule. SIAM J Control Optim, 2001, 40: 64-87

14 Anderson W J. Continuous-Time Markov Chains. New York: Springer, 1991

15 Ji Y, Chizeck H J, Feng X, et al. Stability and control of discrete-time jump linear systems. Contr Theor Adv Tech, 1991, 7: 247-270

16 Mariton M. Jump Linear Systems in Automatic Control. New York: Marcel Dekker, 1990

17 Skorohod A V. Asymptotic Methods in the Theory of Stochastic Differential Equations. Providence: American Mathematical Society, 1989

18 Yin G, Zhang Q. Continuous-Time Markov Chains and Applications: a Singular Perturbation Approach. New York: Springer-Verlag, 1998

19 Shaikhet L. Stability of stochastic hereditary systems with Markov switching. Theory Stoch Proc, 1996, 2: 180-183

20 Mao X R. Stability of stochastic differential equations with Markovian switching. Stochastic Processes Their Appl, 1999, 79: 45-67

21 Arnold L, Crauel H, Wihstutz V. Stabilization of linear systems by noise. SIAM J Control Optim, 1983, 21: 451-461

22 Basak G K, Bisi A, Ghosh M K. Stability of a random diffusion with linear drift. J Math Anal Appl, 1996, 202: $604-622$

23 Khasminskii R Z. Stochastic Stability of Differential Equations. Leiden: Sijthoff and Noordhoff, 1981

24 Mao X R. Stochastic stabilization and destabilization. Syst Control Lett, 1994, 23: 279-290

25 Mao X R. Stochastic Differential Equations and Their Applications. 2nd ed. Chichester: Horwood Publishing Limited, 
2007

26 Appleby J A D, Mao X R. Stochastic stabilisation of functional differential equations. Syst Control Lett, 2005, 54: 1069-1081

27 Mao X R, Yuan C G. Stochastic Differential Equations With Markovian Switching. London: Imperial College Press, 2006

28 Mao X R, Yin G G, Yuan C G. Stabilization and destabilization of hybrid systems of stochastic differential equations. Automatica, 2007, 43: 264-273

29 Scheutzow M K R. Stabilization and destabilization by noise in the plane. Stochastic Anal Appl, 1993, 11: 97-113

30 Mao X R. Stabilization of continuous-time hybrid stochastic differential equations by discrete-time feedback control. Automatica, 2013, 49: 3677-3681

31 Mao X R, Liu W, Hu L J, et al. Stabilization of hybrid stochastic differential equations by feedback control based on discrete-time state observations. Syst Control Lett, 2014, 73: 88-95

32 You S, Liu W, Lu J, et al. Stabilization of hybrid systems by feedback control based on discrete-time state observations. SIAM J Control Optim, 2015, 53: 905-925

33 Song G, Zheng B C, Luo Q, et al. Stabilisation of hybrid stochastic differential equations by feedback control based on discrete-time observations of state and mode. IET Control Theor Appl, 2017, 11: 301-307

34 Mao X R. Almost sure exponential stabilization by discrete-time stochastic feedback control. IEEE Trans Automat Contr, 2016, 61: 1619-1624

\section{Appendix A}

Once Assumption 2 holds, the verification of Assumption 3 depends very much on the choice of $p \in(0,1)$. In this appendix, we give some easier qualifications that guarantee the existence of such a $p$ and, hence, for Assumption 3 to hold. For this purpose, we need the theory of $M$-matrices (see $[2,27]$ ). For a vector or matrix $A, A>0$ means that all elements of $A$ are positive. Moreover, a square matrix $A=\left[a_{i j}\right]_{N \times N}$ is called a $Z$-matrix if it has nonpositive off-diagonal entries, namely

$$
a_{i j} \leqslant 0 \text { for all } i \neq j \text {. }
$$

Here, we cite a useful lemma on $M$-matrices.

Lemma $\mathbf{A 1}$ ([20]). If $A=\left[a_{i j}\right]_{N \times N}$ is a $Z$-matrix, the following statements are equivalent:

(1) $A$ is a nonsingular $M$-matrix.

(2) $A$ is semi-positive; that is, there exists $x>0$ in $\mathbb{R}^{N}$ such that $A x>0$.

(3) $A^{-1}$ exists and its elements are all nonnegative.

(4) All the leading principal minors of $A$ are positive; that is

$$
\left|\begin{array}{ccc}
a_{11} & \cdots & a_{1 k} \\
\vdots & & \vdots \\
a_{k 1} & \cdots & a_{k k}
\end{array}\right|>0, \quad \text { for every } k=1,2, \ldots, N .
$$

We also need another classical result.

Lemma A2 $\left(\right.$ Minkowski $\left.^{1)}\right)$. If a $Z$-matrix $A=\left[a_{i j}\right]_{N \times N}$ has all positive row sums, that is

$$
\sum_{j=1}^{N} a_{i j}>0, \quad \forall i=1,2, \ldots, N
$$

$\operatorname{det} A>0$.

Now, let us propose a condition that

$$
\left|\begin{array}{cccc}
-\left(\alpha_{1}+0.5 \rho_{1}^{2}-\sigma_{1}^{2}\right) & -\gamma_{12} & \cdots & -\gamma_{1 N} \\
-\left(\alpha_{2}+0.5 \rho_{2}^{2}-\sigma_{2}^{2}\right) & -\gamma_{22} & \cdots & -\gamma_{2 N} \\
\vdots & \vdots & \cdots & \vdots \\
-\left(\alpha_{N}+0.5 \rho_{N}^{2}-\sigma_{N}^{2}\right) & -\gamma_{N 2} & \cdots & -\gamma_{N N}
\end{array}\right|>0
$$

where $\alpha_{i}, \rho_{i}, \sigma_{i}$ are the constants specified in Assumption 2. This condition can be verified straightaway once Assumption 2 holds.

It was shown in [28] that under an additional condition that

$$
\text { for some } u \in \mathbb{S}, \gamma_{i u}>0 \text {, for all } i \neq u \text {, }
$$

condition (A1) is equivalent to the following simpler condition

$$
\sum_{i=1}^{N} \pi_{i}\left(\alpha_{i}+0.5 \rho_{i}^{2}-\sigma_{i}^{2}\right)<0,
$$

1) Minkowski H. Diophantische Approximationen. Teubner, 1907. 
where $\left(\pi_{1}, \ldots, \pi_{N}\right)$ are the stationary distribution of the Markov chain as defined in Section 2. In this paper, we replace condition (A2) by a slightly weaker one that

$$
\text { for some } u \in \mathbb{S}, \gamma_{i u} \vee\left(\sigma_{i}^{2}-0.5 \rho_{i}^{2}-\alpha_{i}\right)>0, \quad \text { for all } i \neq u \text {. }
$$

We do not yet know whether (A1) is equivalent to (A3) under this weaker condition. However, the following proposition is good enough for use in this paper.

Proposition A1. If conditions (A1) and (A4) hold, Assumption 3 is satisfied.

Proof. Without loss of generality, we may assume that the state $u=N$ in condition (A4), namely

$$
\gamma_{i N} \vee\left(\sigma_{i}^{2}-0.5 \rho_{i}^{2}-\alpha_{i}\right)>0, \quad \text { for all } 1 \leqslant i \leqslant N-1 .
$$

If not, by switching state $u$ with $N$, we need to reorder the states of the Markov chain $r(t)$ that is, rename state $u$ as $N$ and $N$ as $u$. Consequently, the determinant in the left-hand side of (A1) will switch the $u$ th row with the $N$ th row and then switch the $u$ th column with the $N$ th column, but these do not change the value of the determinant, namely the determinant remains positive. Moreover, given a nonsingular $M$-matrix, it is easy to show that the new matrix remains a nonsingular $M$-matrix by switching the $u$ th column with the $N$ th column and then switching the $u$ th row with the $N$ th row.

By $[27$, Lemma 5.2], the derivative $\mathrm{d} \mathcal{A}(0) / \mathrm{d} p$ is equal to the determinant on the left-hand side of (A1), whence $\mathrm{d} \mathcal{A}(0) / \mathrm{d} p>$ 0 . It is also easy to see $\mathcal{A}(0)=0$. Consequently, for all $p \in(0,1)$ sufficiently small, we have

$$
\operatorname{det} \mathcal{A}(p)>0 \text {. }
$$

On the other hand, for each $i=1,2, \ldots, N-1$, either $\gamma_{i N}>0$ or $\gamma_{i N}=0$. In the case when $\gamma_{i N}>0$, we clearly have

$$
\theta_{i}(p)>-\gamma_{i N}, \quad \text { for all sufficiently small } p \in(0,1) \text {; }
$$

whereas in the case when $\gamma_{i N}=0$, condition (A5) implies $\sigma_{i}^{2}-0.5 \rho_{i}^{2}-\alpha_{i}>0$ whence

$$
\theta_{i}(p)>0=-\gamma_{i N}, \quad \text { for all sufficiently small } p \in(0,1) .
$$

In other words, we always have

$$
\theta_{i}(p)>-\gamma_{i N}, \quad i=1,2, \ldots, N-1,
$$

for all $p \in(0,1)$ sufficiently small. Fix any $p \in(0,1)$ sufficiently small for both (A6) and (A7) to hold. Consider the deriving principal sub-matrix,

$$
\mathcal{A}_{k}(p):=\left[\begin{array}{cccc}
\theta_{1}(p)-\gamma_{11} & -\gamma_{12} & \cdots & -\gamma_{1 k} \\
-\gamma_{21} & \theta_{1}(p)-\gamma_{22} & \cdots & -\gamma_{2 k} \\
\vdots & \vdots & \cdots & \vdots \\
-\gamma_{k 1} & -\gamma_{k 2} & \cdots & \theta_{k}(p)-\gamma_{k k}
\end{array}\right]
$$

of $\mathcal{A}(p)$, for $k=1,2, \ldots, N-1$. Obviously, $\mathcal{A}_{k}(p)$ is a $Z$-matrix. Moreover, for every $i=1,2, \ldots, k$, the $i$ th row of this sub-matrix has its sum

$$
\theta_{i}(p)-\sum_{j=1}^{k} \gamma_{i j}=\theta_{i}(p)+\sum_{j=k+1}^{N} \gamma_{i j} \geqslant \theta_{i}(p)+\gamma_{i N}>0
$$

by (A7). By Lemma A2, $\operatorname{det} \mathcal{A}_{k}(p)>0$. Thus, we should point out that all the deriving principal minors of $\mathcal{A}(p)$ are positive. By applying Lemma $\mathrm{A} 1$, we can obtain that $\mathcal{A}(p)$ is a nonsingular $M$-matrix. This completes the proof. 